\title{
A Novel Serum MicroRNA Panel To Discriminate Benign From Malignant Ovarian Disease
}

Ream Langhe ${ }^{1,2}$, Lucy Norris ${ }^{1}$, Feras Abu Saadeh ${ }^{3}$, Gordon Blackshields ${ }^{2,}$ Rachel Varley ${ }^{1}$, Ashling Harrison ${ }^{1}$, Noreen Gleeson ${ }^{3}$, Cathy Spillane ${ }^{4}$, Cara Martin ${ }^{4}$, Dearbhaile M O' Donnell ${ }^{5}$, Tom D' Arcy ${ }^{3}$, John O'Leary ${ }^{2,4^{*}}$, Sharon O'Toole ${ }^{1,2^{*}}$.

${ }^{1}$ Department of Obstetrics and Gynaecology, Trinity College Dublin, St. James's Hospital, Dublin 8, Ireland

${ }^{2}$ Department of Histopathology, Trinity College Dublin, St. James's Hospital, Dublin 8, Ireland ${ }^{3}$ Department of Gynaecological-Oncology, St. James's Hospital, Dublin 8, Ireland

${ }^{4}$ Department of Histopathology, Trinity College Dublin, Coombe Women's and Infants University Hospital, Dublin 8, Ireland

${ }^{5}$ Department of Medical Oncology, St. James's Hospital, Dublin 8, Ireland

*Authors contributed equally to this project.

\section{Corresponding author:}

Dr. R. Langhe

Postal Address: Department of Obstetrics \& Gynaecology, Trinity Centre for Health Sciences, St. James's Hospital, Dublin 8, Ireland.

E-mail: reamlanghe@yahoo.co.uk

Telephone: 00353871408409

Fax: $\quad 0035314531614$ 


\begin{abstract}
Background: Ovarian cancer is the seventh most common cancer in women and the most frequent cause of gynaecological malignancy-related mortality in women. Currently, no standardized reliable screening test exists. MicroRNA profiling has allowed the identification of signatures associated with diagnosis, prognosis and response to treatment of human tumours. The aim of this study was to determine if a microRNA signature could distinguish between malignant and benign ovarian disease.
\end{abstract}

Methods: A training set of 5 serous ovarian carcinomas and 5 benign serous cystadenomas were selected for the initial experiments. The validation set included 20 serous ovarian carcinomas and 20 benign serous cystadenomas. The serum/plasma focus microRNA Exiqon panel was used for the training set. For the validation set a pick and mix Exiqon panel, which focuses on microRNAs of interest was used.

Results: A panel of 4 microRNAs (let-7i-5p, miR-122, miR-152-5p and miR-25-3p) was significantly down regulated in cancer patients. These microRNAs target WNT signaling, AKT/mTOR and TLR-4/MyD88, which have previously been found to play a role in ovarian carcinogenesis and chemo resistance.

Conclusion: let-7i-5p, miR-122, miR-152-5p and miR-25-3p could act as diagnostic biomarkers in ovarian cancer.

Key words

MicroRNAs, serous ovarian cancer 


\section{Introduction}

Ovarian cancer is the seventh most common cancer in women worldwide with 239,000 new cases diagnosed in 2012 [1]. The incidence rates of the disease vary significantly across different regions of the world. In 2012 the rate of ovarian cancer was more than two times higher in Central and Eastern Europe compared with Eastern Asia [1]. The vast majority of women present in advanced stages and this is due to vagueness of symptoms. Early diagnosis is key as the 5 year survival rate for women diagnosed with late stage disease is less than $20 \%$ compared to up to $90 \%$ for women diagnosed at early stage disease [2-4].

Currently, no established screening program exists to reliably detect ovarian cancer. However, results are awaited from the UK screening trial, UKCTOCS in 2015. The only biomarker that is widely used in clinical practice is CA125 [5]. CA125 is found at abnormally high levels in $80 \%$ of patients with advanced stage disease. However, it is elevated in only $50 \%$ of women with early stage ovarian cancer.

Recently, microRNAs; new biomarkers with diagnostic implications have been identified [6, 7]. MicroRNAs are endogenous non-coding RNA sequences of about 22 nucleotides, which processed from 70-100 nucleotide hairpin pre-microRNAs [8-10]. MicroRNAs inhibit gene expression by inducing degradation or repressing translation of mRNAs when the nucleotide sequences of microRNAs are entirely or partially complementary to the 3'-untranslated regions of targeted mRNAs [11-13].

An increasing number of studies have revealed the vital role microRNAs play in ovarian cancer carcinogenesis [14-21]. However, Most of these studies have been performed on ovarian tissue samples. Recent studies have demonstrated that microRNAs are circulating freely in serum and other body fluids in a highly stable, cell-free form [22-29]. Circulating microRNAs are highly tissue specific that can identify origin of metastasis and can be used as non-invasive biomarkers for cancers $[22,30,31]$. Several studies reported the association of circulating microRNAs with ovarian cancer detection, staging, grading of the disease, outcome and overall survival [32-35]. 
While microRNAs are abundant in tissues, they are often scarce in plasma and serum. For quantification of microRNA in plasma it is therefore essential to use a platform with high sensitivity and linear performance in the low concentration range. The performance of Exiqon and other available microRNA quantification platforms has been evaluated in one study. The reproducibility and sensitivity of Exiqon and other platforms was good and comparable for samples with abundant microRNAs. However, for the analysis of samples with low microRNAs such as serum and plasma, the miRCURY platform was significantly more sensitive than the other platforms [36]. Moreover, Exiqon platform developed a set of quality control for monitoring of haemolysis, which was not been addressed by other platforms. In this study we profiled microRNAs from serum of patients diagnosed with ovarian cancer and benign ovarian disease using Exiqon platform, which takes all of these factors into consideration. Our objectives were to assess the utility of microRNAs as diagnostic biomarkers for ovarian cancer and to determine if microRNAs could distinguish malignant from benign ovarian lesions.

\section{Material and Methods}

\subsection{Samples}

Peripheral blood samples were collected in St. James's Hospital. Ethical approval was obtained from St. James's Hospital and Adelaide and Meath Hospital, Dublin Incorporating the National Children's hospital research Ethics Committee.

Whole blood collected in VACUETTE® tubes was centrifuged at $7500 \mathrm{rpm}$ for 10 minutes. Serum was removed, aliquoted and stored at $-80^{\circ} \mathrm{C}$.

\subsection{RNA isolation}

Serum was thawed on ice and centrifuged at $3000 \mathrm{~g}$ for $5 \mathrm{~min}$ in a $4{ }^{\circ} \mathrm{C}$ microcentrifuge. Total RNA was extracted from serum using the Qiagen miRNeasy® Mini Kit according to manufacturer's instruction. The RNA was stored at $-80{ }^{\circ} \mathrm{C}$. 


\section{3 microRNA real-time $q P C R$}

RNA was reverse transcribed using the miRCURY Locked Nucleic Acid (LNA ${ }^{\mathrm{TM}}$ ) Universal Reverse Transcription (RT) microRNA PCR, Polyadenylation and cDNA synthesis kit-Exiqon. cDNA was diluted $50 \mathrm{x}$ and assayed in $10 \mathrm{ul}$ PCR reactions according to the protocol; each microRNA was assayed once by qPCR on the microRNA Ready-to-Use PCR serum/plasma panel for the pilot study and custom Pick-\&-Mix for the validation study. Negative controls excluding template from the reverse transcription reaction was performed and profiled like the samples.

The amplification was performed in a LightCycler® 480 Real-Time PCR System (Roche) in 384-well plates. The amplification curves were analyzed using the Roche LightCycler software, both for determination of $\mathrm{Cp}$ (or $\mathrm{Ct}$ depends on PCR machine) (by the 2nd derivative method) and for melting curve analysis.

5 serous ovarian carcinomas and 5 benign serous cystadenomas were selected for the pilot study. A validation study included 20 serous ovarian carcinomas and 20 benign serous cystadenomas. The characteristics of study subjects are given in Table 1 .

\subsection{Data quality control}

\subsubsection{Signals from negative controls}

The data set that passed the quality controls was compared to a blank purification sample; negative control (No Template Control, NTC), which was included in the RT step. NTC detects RNA contamination in the RT step. An assay is required to be detected $5 \mathrm{Cp}$ 's lower than the negative control to include it in the data analysis. For assays that do not yield any signal on the negative control, the upper limit of detection is set to $\mathrm{Cp}=37$

\subsubsection{Spike-ins (technical controls)}

An RNA spike-in kit for quality control of the cDNA synthesis has been applied. The cDNA synthesis control (UniSp6) was added in the reverse transcription reaction giving the opportunity to evaluate the RT reaction. In addition to this a DNA spike-in (Sp3) was present in triplicate on all panels. The DNA spike-in consists of a premixed combination of DNA template and primers. Deviations in this reaction will indicate 
inhibitions at the qPCR level.

\subsubsection{Assessing hemolysis}

To assess hemolysis two microRNAs are used; miRNA-451a that is expressed in red blood cells and miRNA-23a-3p that is relatively stable in serum and plasma and not affected by hemolysis. The $\mathrm{dCp}$ value of $\mathrm{miR}-23 \mathrm{a}-3 \mathrm{p} / \mathrm{miR}-451 \mathrm{a}$ was calculated. The ratio between these two microRNAs correlates to degree of hemolysis. Samples with ratios above 8.0 will have an increased risk of being affected by hemolysis. Samples with lower ratios are generally not affected by hemolysis.

\subsection{Data analysis}

The amplification efficiency was calculated using algorithms similar to the LinRegPCR software. All assays were inspected for distinct melting curves and the melting temperature (Tm) was checked to be within known specifications for the assay. Furthermore assays must be detected with 5 Cp's less than the negative control, and with $\mathrm{Cp}<37$ to be included in the data analysis. Data that did not pass these criteria were omitted from any further analysis.

\subsection{Normalization}

Normalization of serum/plasma microRNA qPCR data is challenging. It adjusts for technical biases (RNA amount, quality etc). Normalization is necessary to make expression values comparable across samples. No housekeeping genes are stably expressed in all situations. With qPCR panels, no prior assumptions are made about housekeeping genes. Normalization is performed based on the average of the assays detected in all samples as this is shown to be the best normalization for qPCR studies involving numerous assays [37]. For the training set, this included 95 assays.

Normalization for the validation set is performed based on the average of the normalizer assays and this included has-miR-103a-3p, miR-27b-3p, miR-30b-5p and miR-101-3p. The stability of the average of 95 microRNAs is higher than any single microRNA in the data set as measured by the normfinder software [37]. The formula used to calculate the normalized $\mathrm{Cp}$ values are:

Normalized $\mathrm{Cp}=$ average $\mathrm{Cp}(\mathrm{n}=10)$-assay $\mathrm{Cp}$ (sample) A higher value thus indicates that the microRNA is more abundant in the particular sample. 


\subsection{Bioinformatics}

Validated targets were identified using the MIRWALK database focusing on validated targets, which were then uploaded into DAVID pathway analysis software. ENTREZ_GENE_ID was selected as a gene identifier and Gene List as the list type. KEGG PATHWAY was chosen to see a list of enriched pathways described in the KEGG database. 


\section{Results}

3.1 Controls (NTC and RNA spike-in) indicated good technical performance of the profiling experiment

Signals from negative controls were compared with data set readings that passed quality control showing there was no contamination in the RT step, hence indicating efficient extraction. (Figure $1 \mathrm{a} \& b$ ).

RNA and DNA spike-in are used as technical controls to check if the technical performance of all samples is similar. The steady level of these assays for both the Sp3 and Sp6 assays indicate that both RT and qPCR were successful (Figure $1 \mathrm{c \& d}$ and Tables $2 \& 3)$.

\subsection{Evaluation for potential hemolysis of the blood samples}

The ratio between miRNA-23a-3p and miRNA-451a, which monitor haemolysis was calculated. The dCp (23a-3p /451a) were lower than 8.0 in all samples showing minimal signs of hemolysis problems (Figure 2 a, Table 4).

\subsection{Number of assays detected in all samples}

Of the 176 assays included in miRCURY LNA ${ }^{\mathrm{TM}}$ Universal RT Serum Panel 95 assays were detected in all samples. Based on the results of the pilot study the top 23 deregulated microRNAs (based on fold changes and p-value) were validated (Table 5). Of the 23 microRNAs included in the panel 8 microRNAs were detected in all samples.

To crudely determine data quality for each sample the number of microRNAs detected as well as the average $\mathrm{Cp}$ for each sample was compared in all samples. In this study the samples were alike in microRNA content, suggesting that the samples were of similar quality and have been processed reproducibly (Figure 2 b\&c).

\section{4 microRNAs are downregulated in the malignant group}

When comparing malignant and benign groups using a Students t-test, five microRNAs were differentially expressed using a cutoff of p-value $<0.05$. Four of these p-values passed a Benjamini Hochberg correction for multiple testing. Table 6 shows the individual results for the top 8 microRNAs. 


\section{5 Hierarchical clustering}

The heat map diagram shows the result of the two-way hierarchical clustering of microRNAs and samples. Each row represents one microRNA, and each column represents one sample. The microRNA-clustering tree is shown on the left. The color scale shown at the bottom illustrates the relative expression level of a microRNA across all samples: red color represents an expression level above mean, green color represents expression lower than the mean.

The top 50 microRNA have been included in the two-way hierarchical clustering of microRNAs (Figure 3). Heat Map and Unsupervised Hierarchical Clustering is performed on all samples, and on the top 50 microRNAs with highest standard deviation. The normalized $(\mathrm{dCp})$ values have been used for the analysis.

\subsection{Bioinformatic analysis of the validated targets for the four-downregulated microRNAs revealed numerous pathways involved in cancer}

Bioinformatic analysis of the deregulated microRNAs in cancer patients in our study demonstrated several pathways. Validated targets were identified using the MIRWALK database. 919 validated targets were identified for the top four microRNAs. 578 validated targets for let-7i-5p, 212 for miR-122-5p, 81 for miR-25$3 p$ and 48 targets for miR-152 have been found.

The validated targets were then uploaded into the DAVID pathway analysis software. KEGG PATHWAY was chosen to see a list of enriched pathways described in the KEGG database. To avoid over counting duplicated genes, the Fisher Exact statistics is calculated based on corresponding DAVID gene IDs by which all redundancies in original IDs are removed. All result of Chart Report has to pass the thresholds (by default, Max.Prob.<=0.1 and Min.Count $>=2$ ) in Chart Option section to ensure only statistically significant ones displayed, the smaller the $\mathrm{p}$ value the more enriched the pathway. Tables 7,8,9,10 and 11(Supplemental) show lists of pathways, which involve the validated targets for differentially deregulated microRNAs in ovarian cancer patients in this study. 
When examined individually the validated targets of the microRNAs were found to be involved in numerous pathways; let-7i-5p in 56 pathways, miR-122-5p in 34 pathways, miR-152 in 20 pathways and miR-25-3p in 34 pathways in ovarian cancer and other cancers such as pancreatic cancer, colorectal cancer and others. When all four microRNAs were examined together to determine common pathways in relation to ovarian cancer the following pathways were revealed, Toll-like receptor signaling, mTOR signaling, and VEGF signaling pathways.

\section{6 microRNAs have prognostic potential}

Patterns of microRNAs were analysed in relation to chemotherapy response. Patients who developed chemoresistance early lost expression of let-7i, which might suggest its utility as a prognostic marker. 


\section{Discussion}

This study successfully profiled and validated differentially regulated microRNAs in serum from ovarian cancer patients versus benign controls using the Exiqon platform and revealed four (let-7i-5p, miR-152, miR-122-5p and miR-25-3p) significantly down-regulated microRNAs in cancer patients.

The majority of miRNA papers published across multiple disease systems to date consider a change of $>1.5$ fold as significant [38-40] and confirm subtle changes in microRNA expression can have a significant and more dramatic knock-on effect for the mRNA/protein expression levels [41, 42].

Circulating microRNAs are rarely more differentially expressed than 2-3 fold. The Exiqon platform used in our study is sensitive enough to reliably detect differences in that range. Recent papers $[43,44]$ that used Exiqon qPCR technology report fold changes in the same range as the current study. So the microRNA community as such accepts that small changes are/can be meaningful.

Exiqon has several advantages compared to other platforms. One of these advantages is adding a carrier RNA during the purification procedure, which ensures the highest and most consistent yield from serum samples. In addition, it minimizes the carryover of enzyme inhibitors, which are present at high levels in serum and that affect the efficiency of the RT or PCR reactions while maximizing the RNA yield.

Furthermore, Exiqon has developed a set of synthetic RNA spike-ins that can be used to perform qPCR-based quality control of RNA samples. Another advantage is monitoring of hemolysis, where the level of miR-451a in red blood cells is compared with miR-23a-3p [45]. Blood cells are a major contributor to circulating microRNAs and perturbations in blood cell counts and hemolysis can alter the plasma levels of microRNA biomarker by up to 50-fold [46]. Measurements of the level of these microRNAs in whole blood, plasma, red blood cells and peripheral blood cells revealed that the microRNA content of red blood cells represents the major source of variation in miR-451a levels measured in plasma [47]. Our study addressed the issue of microRNA abundance arising from haemolysed red blood cells, which was not addressed by previous serum studies. 
Our study has many advantages over previous studies. Firstly, we used benign disease as a control group compared to most studies, which used healthy women as a control group. As ovarian cancer affects postmenopausal women, it was very difficult to get age matched healthy control; furthermore healthy women need to be followed to ensure absence of other disease. In addition, many of the microRNAs published as being associated with ovarian cancer were found in our group so most likely they are related to the benign tumorigenesis process and not malignant disease. Also the malignant group was confined to the most common and lethal histological subtype high-grade serous cancers.

Let-7i was the most significant down-regulated microRNA in our study. It has been extensively researched in ovarian cancer and was significantly decreased in chemotherapy-resistant patients [48]. Reduced let-7i expression in tissue was significantly associated with shorter progression-free survival of patients who presented with advanced ovarian cancer. These results strongly indicate that let-7i might be used as a therapeutic target to modify platinum-based chemotherapy and as a biomarker to predict response to chemotherapy and survival in patients with ovarian cancer [48]. Loss of let-7i was found to correlate with intrinsic chemoresistance, which might suggest its role, as a triage marker for chemotherapy and this would need to be evaluated in a larger cohort.

The TLR4 gene is a validated target of the let-7i miRNA and our group has just recently demonstrated that overexpression of TLR4/MyD88 is an adverse prognostic marker in ovarian cancer [49]. We have shown that overexpression of TLR4 and MyD88 results in a shorter progression free survival and overexpression of MyD88 in a shorter overall survival. Of interest in our cohort this miRNA disappeared in those patients who developed chemoresistance early which further strengthens the evidence for the importance of this miRNA in ovarian cancer.

Let-7i has been linked to invasion, one of the key hallmarks of cancer that differentiates benign and malignant cases. One study revealed a role for Let-7i in the initiation and malignant progression of locally advanced gastric cancer (LAGC) and its possible value in prediction of neo-adjuvant chemotherapeutic efficacy [50]. 
Reduced let-7i expression was an unfavorable prognostic factor of overall survival independently of other clinic-pathological factors, such as tumor node metastasis and depth of infiltration [50]. These results suggest that let-7i might act as a useful therapeutic target and a potential prognostic biomarker in LAGC patients [50] and it may play a similar role in ovarian cancer. Our study is the first to report serum let-7i in the context of a cancer biomarker.

miR-122 has not been reported in ovarian cancer previously but it has been associated with other cancers such as breast cancer. miR-122 was reported to serve as a tumour suppressor and has a vital role in inhibiting breast cancer tumorigenesis through targeting IGF1R and regulating the PI3K/Akt/mTOR/p70S6K pathway. These findings indicate that miR-122 may be a novel therapeutic or diagnostic/prognostictarget for treating breast cancer [51] and may play a similar role in ovarian cancer, which requires further investigation.

miR-152 was significantly reduced in ovarian cancer tissues compared to normal ovarian epithelium tissues [52]. Overexpression of miR-152 and miR-185 enhanced cisplatin sensitivity by inhibiting proliferation and accelerating apoptosis through direct targeting of DNA methyltransferase 1 (DNMT1) tumour angiogenesis and increasing vascular permeability. [53].

miR-152 has also been linked to invasion; it was significantly decreased in primary prostate cancer compared to that in non-malignant samples [54]. The potential of miR-152 as a tissue-based biomarker has been demonstrated in many studies described above and our study now for the first time demonstrates its utility as a serum based diagnostic biomarker.

miR-25 is another microRNA, which was highly expressed in ovarian cancer samples and cell lines [55]. In this study apoptosis was induced by down-regulation of miR-25 in ovarian cancer cells, while overexpression of miR-25 enhanced cell proliferation. There was an inverse relationship between the pro-apoptotic protein Bim and expression of miR-25 expression in ovarian cancer tissues. These results suggested that miR-25 directly regulates apoptosis through targeting Bim in ovarian cancer and miR-25 may serve as a potential therapeutic target for ovarian cancer [55].

When carry out pathway analysis on each microRNA individually a number of 
pathways were discovered such as Toll-like receptor (TLR) and mTOR signaling pathways for let-7i-5p, p53 pathway and TLR for miR-122-5p and miR-25-3p and TLR for miR-152. Analysis of the validated targets for the four differentially regulated microRNAs together revealed numerous pathways involved in cancer such as WNT signaling, AKT/mTOR, VEGF and TLR-4/MyD88 signaling pathway. WNT signaling pathway has previously been found to play a role in ovarian carcinogenesis [56].

Another pathway is the AKT/mTOR signaling, which is frequently overexpressed in epithelial ovarian cancer and was targeted by validated targets of let-7i-5p and miR122-5p. In vivo administration of mTOR inhibitors in ovarian cancer xenografts is associated with antitumoral and antiangiogenic effects [57]. Currently mammalian target of rapamycin (mTOR) inhibitors, poly-ADP-ribose polymerase (PARP) inhibitors and components of the EGFR pathway are in the focus of clinical research [58].

VEGF is a key factor involved in the development of ovarian cancer by enhancing validated targets of miR-122-5p. VEGF levels have been inversely correlated with survival. Furthermore, inhibition of VEGF found to suppress tumor invasion and metastasis [59].

TLR-4/MyD88, which was targeted by validated targets for the four microRNAs, has in recent years been proposed as a risk factor for carcinogenesis and chemoresistance in ovarian cancer $[60,61]$ and as discussed above our group and others have demonstrated its importance.

While it has been observed that TLR-4 expression is ubiquitous in Epithelial Ovarian Cancer (EOC) cells, a subgroup differentially expressing MyD88 has demonstrated increased cytokine/chemokine production and cellular proliferation upon activation of TLR-4 [60]. More recently MyD88 protein expression was shown to be a significantly poor factor in EOC by our group and others [49, 62].

\section{Conclusion}

Four microRNAs were significantly downregulated in cancer patients. The validated targets for the significantly differentially expressed microRNAs revealed numerous 
pathways involved in cancer such as WNT signalling, AKT/mTOR, TLR-4/MyD88 and VEGF signaling pathway.

Clearly our identified microRNAs have shown some potential as serum biomarkers in other cancers and some have already shown important roles in the biology of ovarian cancer including their invasive potential, which is one of the key hallmarks that segregates benign and malignant tumours. Discriminating malignant from benign disease has important clinical implications such as less radical treatment for benign lesions, preservation of fertility in young women and reserving waiting lists in gynaecological cancer centers for malignant cases. A prospective validation study is warranted to evaluate the clinical performance of the panel.

\section{Conflict of interest}

The authors declare that they have no competing interests.

\section{Acknowledgment}

We thank theatre staff and gynaecologic oncology team in St James's Hospital. 


\section{List of Figures and Tables}

Figure 1 (a) Raw Cp values for the negative control sample for the pilot study

(b) Raw $\mathrm{Cp}$ values for the negative control sample for the validation study

(c) Raw data for the RNA spike-in, DNA spike-in for the pilot study

(d) Raw data for the UniSP6 spike-in for the validation study

Figure 2 (a) dCp (23a-3p/451a) ratio from the samples analyzed

(b) Number of detected microRNAs and the average Cp for each sample in the pilot study

(c) Number of detected microRNAs and the average $\mathrm{Cp}$ for each sample in the validation study

Figure 3 Heat Map and Unsupervised Hierarchical Clustering for

(a) Pilot study

(b) Validation study

Table 1 Characteristic of the population enrolled in the study

Table 2 Raw data for the RNA and DNA spike in the pilot study

Table 3 Raw data for the RNA and DNA spike in the validation study

Table 4 Raw data for miR-23a-3p \&miR-451a to assess the degree of haemolysis

Table 5 Deregulated microRNAs from the pilot study

Table 6 Differentially expressed microRNAs in the validation study

Supplemental Table 7 List of pathways, which involve the validated targets for let-7i$5 \mathrm{p}$

Supplemental Table 8 List of pathways, which involve the validated targets for miR$122-5 p$

Supplemental Table 9 List of pathways, which involve the validated targets for miR152

Supplemental Table 10 List of pathways, which involve the validated targets for miR$25-3 p$

Supplemental Table 11 List of pathways, which involve the validated targets for the four microRNAs together 


\section{References:}

1. Ferlay J, S.I., Ervik M, Dikshit R, Eser S, Mathers C, Rebelo M, Parkin DM, Forman D, Bray, F. Cancer Incidence and Mortality Worldwide. 2013.

2. Urban, N. and C. Drescher, Potential and limitations in early diagnosis of ovarian cancer. Adv Exp Med Biol, 2008. 622: p. 3-14.

3. Badgwell, D. and R.C. Bast, Jr., Early detection of ovarian cancer. Dis Markers, 2007. 23(5-6): p. 397-410.

4. Goff, B.A., et al., Ovarian carcinoma diagnosis. Cancer, 2000. 89(10): p. 2068-75.

5. Skates, S.J., I.J. Jacobs, and R.C. Knapp, Tumor markers in screening for ovarian cancer. Methods Mol Med, 2001. 39: p. 61-73.

6. Iorio, M.V., et al., MicroRNA signatures in human ovarian cancer. Cancer Res, 2007. 67(18): p. 8699-707.

7. Calin, G.A. and C.M. Croce, MicroRNA-cancer connection: the beginning of a new tale. Cancer Res, 2006. 66(15): p. 7390-4.

8. Bartel, D.P., MicroRNAs: genomics, biogenesis, mechanism, and function. Cell, 2004. 116(2): p. 281-97.

9. Lagos-Quintana, M., et al., Identification of novel genes coding for small expressed RNAs. Science, 2001. 294(5543): p. 853-8.

10. Lau, N.C., et al., An abundant class of tiny RNAs with probable regulatory roles in Caenorhabditis elegans. Science, 2001. 294(5543): p. 858-62.

11. Miska, E.A., How microRNAs control cell division, differentiation and death. Curr Opin Genet Dev, 2005. 15(5): p. 563-8.

12. Esquela-Kerscher, A. and F.J. Slack, Oncomirs - microRNAs with a role in cancer. Nat Rev Cancer, 2006. 6(4): p. 259-69.

13. Gregory, R.I. and R. Shiekhattar, MicroRNA biogenesis and cancer. Cancer Res, 2005. 65(9): p. 3509-12.

14. Vang, S., et al., Identification of Ovarian Cancer Metastatic miRNAs. PLoS One, 2013. 8(3): p. e58226.

15. Chung, Y.W., et al., Detection of MicroRNA as Novel Biomarkers of Epithelial Ovarian Cancer From the Serum of Ovarian Cancer Patient. Int J Gynecol Cancer, 2013. 23(4): p. 673-9.

16. Qin, W., et al., MicroRNA-155 is a novel suppressor of ovarian cancerinitiating cells that targets CLDN1. FEBS Lett, 2013.

17. He, J., et al., Roles and mechanism of miR-199a and miR-125b in tumor angiogenesis. PLoS One, 2013. 8(2): p. e56647.

18. Gallagher, M.F., et al., Regulation of microRNA biosynthesis and expression in 2102Ep embryonal carcinoma stem cells is mirrored in ovarian serous adenocarcinoma patients. J Ovarian Res, 2009. 2: p. 19.

19. Flavin, R., et al., miR-29b expression is associated with disease-free survival in patients with ovarian serous carcinoma. Int J Gynecol Cancer, 2009. 19(4): p. 641-7.

20. Flavin, R.J., et al., Potentially important microRNA cluster on chromosome 17p13.1 in primary peritoneal carcinoma. Mod Pathol, 2009. 22(2): p. 197205.

21. Laios, A., et al., Potential role of miR-9 and miR-223 in recurrent ovarian cancer. Mol Cancer, 2008. 7: p. 35. 
22. Mitchell, P.S., et al., Circulating microRNAs as stable blood-based markers for cancer detection. Proc Natl Acad Sci U S A, 2008. 105(30): p. 10513-8.

23. Chen, X., et al., Characterization of microRNAs in serum: a novel class of biomarkers for diagnosis of cancer and other diseases. Cell Res, 2008. 18(10): p. 997-1006.

24. Chim, S.S., et al., Detection and characterization of placental microRNAs in maternal plasma. Clin Chem, 2008. 54(3): p. 482-90.

25. Gilad, S., et al., Serum microRNAs are promising novel biomarkers. PLoS One, 2008. 3(9): p. e3148.

26. Lawrie, C.H., et al., Detection of elevated levels of tumour-associated microRNAs in serum of patients with diffuse large B-cell lymphoma. Br J Haematol, 2008. 141(5): p. 672-5.

27. Taylor, D.D. and C. Gercel-Taylor, MicroRNA signatures of tumor-derived exosomes as diagnostic biomarkers of ovarian cancer. Gynecol Oncol, 2008. 110(1): p. 13-21.

28. Hunter, M.P., et al., Detection of microRNA expression in human peripheral blood microvesicles. PLoS One, 2008. 3(11): p. e3694.

29. Shapira, I., et al., Circulating biomarkers for detection of ovarian cancer and predicting cancer outcomes. Br J Cancer, 2014. 110(4): p. 976-83.

30. Cheng, H., et al., microRNA signature for human pancreatic cancer invasion and metastasis. Exp Ther Med, 2012. 4(2): p. 181-187.

31. Chen, X., et al., Characterization of microRNAs in serum: a novel class of biomarkers for diagnosis of cancer and other diseases. Cell Res, 2008. 18(10): p. 997-1006.

32. Resnick, K.E., et al., The detection of differentially expressed microRNAs from the serum of ovarian cancer patients using a novel real-time PCR platform. Gynecol Oncol, 2009. 112(1): p. 55-9.

33. Guo, F., et al., Serum microRNA-92 expression in patients with ovarian epithelial carcinoma. J Int Med Res, 2013. 41(5): p. 1456-61.

34. Xu, Y.Z., et al., Identification of Serum MicroRNA-21 as a Biomarker for Early Detection and Prognosis in Human Epithelial Ovarian Cancer. Asian Pac J Cancer Prev, 2013. 14(2): p. 1057-60.

35. Hong, F., et al., Prognostic significance of serum microRNA-221 expression in human epithelial ovarian cancer. J Int Med Res, 2013. 41(1): p. 64-71.

36. Jensen, S.G., et al., Evaluation of two commercial global miRNA expression profiling platforms for detection of less abundant miRNAs. BMC Genomics, 2011. 12: p. 435.

37. Andersen, C.L., J.L. Jensen, and T.F. Orntoft, Normalization of real-time quantitative reverse transcription-PCR data: a model-based variance estimation approach to identify genes suited for normalization, applied to bladder and colon cancer data sets. Cancer Res, 2004. 64(15): p. 5245-50.

38. Backes, C., et al., Blood born miRNAs signatures that can serve as disease specific biomarkers are not significantly affected by overall fitness and exercise. PLoS One, 2014. 9(7): p. e102183.

39. Leidinger, P., et al., The blood-borne miRNA signature of lung cancer patients is independent of histology but influenced by metastases. Mol Cancer, 2014. 13: p. 202.

40. Pradervand, S., et al., Impact of normalization on miRNA microarray expression profiling. RNA, 2009. 15(3): p. 493-501. 
41. Ohlsson Teague, E.M., et al., MicroRNA-regulated pathways associated with endometriosis. Mol Endocrinol, 2009. 23(2): p. 265-75.

42. Chakrabarty, A., et al., MicroRNA regulation of cyclooxygenase-2 during embryo implantation. Proc Natl Acad Sci U S A, 2007. 104(38): p. 15144-9.

43. Kodahl, A.R., et al., Alterations in circulating miRNA levels following earlystage estrogen receptor-positive breast cancer resection in postmenopausal women. PLoS One, 2014. 9(7): p. e101950.

44. Sorensen, S.S., et al., miRNA Expression Profiles in Cerebrospinal Fluid and Blood of Patients with Acute Ischemic Stroke. Transl Stroke Res, 2014.

45. Blondal, T., et al., Assessing sample and miRNA profile quality in serum and plasma or other biofluids. Methods, 2013. 59(1): p. S1-6.

46. Pritchard, C.C., et al., Blood cell origin of circulating microRNAs: a cautionary note for cancer biomarker studies. Cancer Prev Res (Phila), 2012. 5(3): p. 492-7.

47. Kirschner, M.B., et al., Haemolysis during sample preparation alters microRNA content of plasma. PLoS One, 2011. 6(9): p. e24145.

48. Yang, N., et al., MicroRNA microarray identifies Let-7i as a novel biomarker and therapeutic target in human epithelial ovarian cancer. Cancer Res, 2008. 68(24): p. 10307-14.

49. D'Adhemar CJ, S.C., Gallagher MF, Bates M, Costello K, Barry O'Crowley J, Haley K, Kernan N, Murphy C, Smyth PC, O’Byrne K, Pennington S, Cooke A, Ffrench B, Martin CM, O'Donnell D, Hennessy B, Stordal B, Finn S, McCann A, Gleeson N, D’Arcy T, Flood B, O'Neill LAJ, Sheils O, O’Toole S*, O'Leary JJ*, The MyD88+ phenotype is an adverse prognostic factor in epithelial ovarian cancer. . PLoS One, 2014.

50. Liu, K., et al., Decreased expression of microRNA let-7i and its association with chemotherapeutic response in human gastric cancer. World J Surg Oncol, 2012. 10: p. 225.

51. Wang, B., H. Wang, and Z. Yang, MiR-122 inhibits cell proliferation and tumorigenesis of breast cancer by targeting IGF1R. PLoS One, 2012. 7(10): p. e47053.

52. Zhou, X., et al., Altered expression of miR-152 and miR-148a in ovarian cancer is related to cell proliferation. Oncol Rep, 2012. 27(2): p. 447-54.

53. Xiang, Y., et al., MiR-152 and miR-185 co-contribute to ovarian cancer cells cisplatin sensitivity by targeting DNMT1 directly: a novel epigenetic therapy independent of decitabine. Oncogene, 2013.

54. Zhu, C., et al., miR-152 controls migration and invasive potential by targeting TGFalpha in prostate cancer cell lines. Prostate, 2013. 73(10): p. 1082-9.

55. Zhang, H., et al., MiR-25 regulates apoptosis by targeting Bim in human ovarian cancer. Oncol Rep, 2012. 27(2): p. 594-8.

56. Gatcliffe, T.A., et al., Wht signaling in ovarian tumorigenesis. Int J Gynecol Cancer, 2008. 18(5): p. 954-62.

57. Trinh, X.B., et al., The rationale for mTOR inhibition in epithelial ovarian cancer. Expert Opin Investig Drugs, 2009. 18(12): p. 1885-91.

58. Smolle, E., et al., Targeting signaling pathways in epithelial ovarian cancer. Int J Mol Sci, 2013. 14(5): p. 9536-55. 
59. Masoumi Moghaddam, S., et al., Significance of vascular endothelial growth factor in growth and peritoneal dissemination of ovarian cancer. Cancer Metastasis Rev, 2012. 31(1-2): p. 143-62.

60. Kelly, M.G., et al., TLR-4 signaling promotes tumor growth and paclitaxel chemoresistance in ovarian cancer. Cancer Res, 2006. 66(7): p. 3859-68.

61. Chen, R., et al., Cancers take their Toll--the function and regulation of Tolllike receptors in cancer cells. Oncogene, 2008. 27(2): p. 225-33.

62. Zhu, Y., et al., Prognostic significance of MyD88 expression by human epithelial ovarian carcinoma cells. J Transl Med, 2012. 10: p. 77. 